Check for updates

Cite this: J. Mater. Chem. A, 2022, 10, 218

Received 19th July 2021

Accepted 5th December 2021

DOI: $10.1039 / d 1 t a 06075 a$

rsc.li/materials-a

\section{Facet-engineered $\mathrm{TiO}_{2}$ nanomaterials reveal the role of water-oxide interactions in surface protonic conduction $\uparrow$}

\author{
Xiaolan Kang, Athanasios Chatzitakis, (D) Thomas Aarholt, Xinwei Sun, Chiara Negri \\ and Truls Norby (D) *
}

Water adsorption and surface protonic conduction have been investigated at $25-400{ }^{\circ} \mathrm{C}$ in wet $\left(\mathrm{H}_{2} \mathrm{O}\right.$ and $\mathrm{D}_{2} \mathrm{O}$ ) atmospheres on nanocrystalline $\mathrm{TiO}_{2}$ hydrothermally grown to a predominance of different crystal facets. In situ Fourier transform infrared spectrometry shows that a portion of the water molecules in the first chemisorbed layer adsorbs dissociatively on the $\{001\}$ and $\{100\}$ surfaces, but molecularly on the $\{101\}$ surface. The next layer of physisorbed molecular water is solid (ice)-like on the $\{001\}$ and $\{100\}$ surfaces, a fact that is attributed to relatively strong bonds to the surface terminating hydroxyls. On the other hand, it is looser, liquid-like on the $\{101\}$ surface due to the lack of rigid hydroxyl groups to bond to. As relative humidity $(\mathrm{RH})$ increases with decreasing temperature, additional water layers physisorb and eventually become liquid-like also on the $\{001\}$ and $\{100\}$ surfaces. The differences can be attributed to the different atomic arrangements and surface energies of the different crystal facets. Surface protonic conduction on $\{001\}$ and $\{100\}$ dominated samples exhibits high pre-exponentials, indicating a high concentration of sites for dissociative chemisorption, and high activation energies reflecting difficult proton migration in the rigid water layers on those surfaces. The surface protonic conduction on the $\{101\}$ dominated sample reflects a lower coverage of dissociable water and lower activation enthalpies for protonic migration in the more loosely bonded water. This suggests that, more generally, surfaces with dissociative chemisorption may exhibit high surface protonic conductivity at the highest and lowest temperatures (corresponding to lowest and highest $\mathrm{RH}$, respectively), while surfaces with molecular chemisorption may exhibit a relatively high conductivity (less deep minimum) at intermediate temperatures. H/D isotope effects show that the protonic conduction mechanism changes from Grotthuss to vehicle as the physisorbed water layers gain thickness and become liquid-like. The protonic conductivity decreased when the surface was terminated by more strongly bonded fluoride ions, believed to reflect that they block the sites for water adsorption.

\section{Introduction}

Surface protonic conduction comprises Grotthuss migration of free protons $\left(\mathrm{H}^{+}\right)$and vehicular migration mainly of hydronium ions $\left(\mathrm{H}_{3} \mathrm{O}^{+}\right)$in chemisorbed and physisorbed water layers on oxides or other materials. ${ }^{1}$ Mesoporous and other nanocrystalline $\mathrm{TiO}_{2}$ materials exhibit high surface protonic conductivity in wet atmospheres, of importance for use as photocatalysts, sensors, and electrodes, and proposedly also as electrolytes in fuel cells. ${ }^{2-4}$ As an electrolyte material, $\mathrm{TiO}_{2}$ cannot compete with the traditional proton exchange membranes (PEM), such as Nafion ${ }^{\circledR}$ that typically operates at temperatures between 80 and $100{ }^{\circ} \mathrm{C}$ with high relative humidity $(\mathrm{RH}),{ }^{5}$ and proton

Centre for Materials Science and Nanotechnology (SMN), University of Oslo, NO-0318 Oslo, Norway. E-mail: truls.norby@kjemi.uio.no

$\dagger$ Electronic supplementary information (ESI) available. See DOI: 10.1039/d1ta06075a conducting ceramics like Y-substituted $\mathrm{BaZrO}_{3}$ (BZY) employed at higher working temperatures $\left(>400{ }^{\circ} \mathrm{C}\right) .{ }^{6}$ However, over a broad range of temperatures between $400{ }^{\circ} \mathrm{C}$ and room temperature, $\mathrm{TiO}_{2}$ nanomaterials exhibit considerable protonic conductivities $^{7,8}$ that in the case of anatase nanofilms have even been reported to exceed that of Nafion ${ }^{\circledR}$ at low temperatures. ${ }^{3}$

Previous studies on the surface protonic conduction of $\mathrm{TiO}_{2}$ based nanomaterials mainly focused on the effects of the specific surface area, pore networks, and grain size, as well as intrinsic or extrinsic dopants. ${ }^{7,9-11}$ For example, Maglia et al. found that the surface protonic conductivity of dense anatase samples with grain sizes between 24 and $56 \mathrm{~nm}$ increased strongly with decreasing grain size. ${ }^{10}$ Stub et al. reported that donor and acceptor doped porous $\mathrm{TiO}_{2}$ materials give rise to increased and decreased surface protonic conductivity, respectively, compared to undoped $\mathrm{TiO}_{2}$. Based on this, they suggested the possible effect of heterovalent doping on the acid-base properties of the $\mathrm{TiO}_{2}$ surface. ${ }^{\mathbf{1 1}}$ The results of the past few years 
imply that the orientation of different crystal facets of $\mathrm{TiO}_{2}$ significantly affects water adsorption and dissociation, ${ }^{\mathbf{1 2}}$ however, to the best of our knowledge, their influence on surface protonic conductivity has not been systematically investigated.

The adsorption of the first layer of water on oxides is generally considered to be the chemisorption of water molecules that bond to terminating cations, followed and stabilized by various degrees of dissociation into adsorbed hydroxyl groups and protons. The latter bonds to terminating surface oxide anions forming another surface hydroxyl group. As $\mathrm{RH}$ increases, a second layer of molecular water physisorbs fairly strongly by hydrogen bonds to the chemisorbed layer underneath. The possibility to make double hydrogen bonds to dissociated hydroxyl groups in a dissociated chemisorbed layer makes it rather crystalline and solid ("ice-like"). At high $\mathrm{RH}$, a few layers more of water molecules physisorb by weaker hydrogen bonds that make them randomly oriented ("liquidlike").

Dissociated protons migrate by Grotthuss-type hopping in the chemisorbed and ice-like physisorbed layers, while at $\mathrm{RH}>$ $0.6(60 \%)$ vehicular transport of $\mathrm{H}_{3} \mathrm{O}^{+}$ions in the liquid-like water takes over. ${ }^{\mathbf{1 1}, \mathbf{1 3}}$

Generally, abundant dangling bonds, such as undercoordinated cations and oxide anions, serve as sites for water adsorption and dissociation, ${ }^{\mathbf{1 2 , 1 4}}$ that can decrease the initial surface energy excess. Anatase $\mathrm{TiO}_{2}$ crystals expose mainly $\{101\},\{001\}$, and $\{100\}$ facets. The most stable $\{101\}$ facet has a saw-tooth-like appearance with half $\mathrm{Ti}_{5 \mathrm{c}}$ (five-fold coordinated $\mathrm{Ti}^{4+}$ cations) and $\mathrm{Ti}_{6 \mathrm{c}}$ atoms as well as half $\mathrm{O}_{2 \mathrm{c}}$ (two-fold coordinated $\mathrm{O}^{2-}$ anions) and $\mathrm{O}_{3 \mathrm{c}}$ atoms, featuring alternating rows of $\mathrm{Ti}_{5 \mathrm{c}}$ and $\mathrm{O}_{2 \mathrm{c}}$ along the [010] direction. ${ }^{15}$ The high surface energy of the $\{001\}$ facet originates mainly from the undercoordinated nature of surface species $\left(\mathrm{Ti}_{5 \mathrm{c}}\right.$ and $\left.\mathrm{O}_{2 \mathrm{c}}\right)$. The $\{100\}$ facet comprises of $\mathrm{Ti}_{5 \mathrm{c}}, \mathrm{O}_{2 \mathrm{c}}$, and $\mathrm{O}_{3 \mathrm{c}}$ atoms and is more stable than the $\{001\}$ facet but less stable than the $\{101\} .{ }^{16}$ Different surface energies, atomic arrangements, and steric hindrance lead to different degrees of molecular or dissociative adsorption characteristics, and we here investigate the effects of these variables on surface protonic conduction.

The extent and configuration of molecularly and dissociatively chemisorbed water on the different facets of anatase $\mathrm{TiO}_{2}$ are debated. For example, Vittadini et al. ${ }^{\mathbf{1 7}}$ found that molecular adsorption on the $\{101\}$ facet is energetically favored over dissociative adsorption by $0.44 \mathrm{eV}$ at low coverage and by $0.28 \mathrm{eV}$ at monolayer coverage. They report that on the $\{001\}$ facet, low coverage is dominated by dissociative adsorption, which turns into mixed molecular/dissociative adsorption at a monolayer coverage. Based on synchrotron radiation core level photoelectron spectroscopy, Walle et al. ${ }^{18}$ proposed mixed molecular and dissociative water adsorption on the $\{101\}$ facet.

In this work, in situ Fourier transform infrared spectrometry (FTIR) has been utilized to study the adsorption, dissociation, and water layer formation as a function of $\mathrm{RH}$ and temperatures on anatase nanocrystallites with predominance of three lowindex facets $\{101\},\{001\}$, and $\{100\}$. Surface protonic conductivity on such facet-engineered porous anatase samples was measured with impedance spectroscopy under controlled temperatures and fixed partial pressure of water. Additionally, the effects of H/D isotope exchange and fluoride ion termination were studied to improve our understanding of the processes underpinning the surface protonic conduction. We model the conductivity in terms of pre-exponentials and activation enthalpies of contributions from bulk, Grotthuss protonic conduction in the chemisorbed and ice-like physisorbed layers, vehicular conduction in the liquid-like layer, and correlate them with adsorption, dissociation, and layer rigidity on the differently terminated surfaces, an approach possibly applicable also to surface protonic conduction of other oxides.

\section{Experimental}

\section{Synthesis of anatase $\mathrm{TiO}_{2}$ nanoparticles with predominance of different facets}

$\mathrm{TiO}_{2}$ nanoparticles with a predominance of the $\{101\}$ facet (T101) were synthesized with a two-step hydrothermal procedure based on previous reports. ${ }^{19}$ First, a precursor of potassium titanate powder with nanotubular morphology was prepared by adding $1 \mathrm{~g}$ of commercial $\mathrm{TiO}_{2}$ powder (P25, SigmaAldrich) to $40 \mathrm{~mL}$ freshly made $17 \mathrm{M} \mathrm{KOH}$ (aq). The mixture was stirred for $20 \mathrm{~min}$ and introduced into a Teflon vessel with an inner volume of $50 \mathrm{~mL}$ for the hydrothermal treatment at $110^{\circ} \mathrm{C}$ for $20 \mathrm{~h}$. The resulting powder was rinsed with dilute $\mathrm{HCl}(\mathrm{aq})$ and deionized water until neutral $(\mathrm{pH} \approx 7)$. In the second step, the as-prepared potassium titanate powder was dispersed in $35 \mathrm{~mL}$ deionized water. The $\mathrm{pH}$ was adjusted to 3.5 with $\mathrm{HCl}$ (aq) during stirring and the mixture was transferred to a $50 \mathrm{~mL}$ Teflon-lined autoclave for the hydrothermal reaction at $200{ }^{\circ} \mathrm{C}$ for $12 \mathrm{~h}$. The T101 powder was finally collected by centrifugation and washed with deionized water.

To synthesize anatase $\mathrm{TiO}_{2}$ with a predominance of the $\{100\}$ facet (T100), a similar procedure was used. In the first hydrothermal process, instead of using $\mathrm{P} 25, \mathrm{TiO}_{2}$ nanoparticles were prepared according to a previously reported method ${ }^{\mathbf{2 0}}$ and used as precursor to synthesize potassium titanate nanotubes. The as-synthesized nanotubes were washed with deionized water, dried at $80{ }^{\circ} \mathrm{C}$. In the second hydrothermal process, $\mathrm{pH}$ was kept neutral.

$\mathrm{TiO}_{2}$ nanosheets with exposed $\{001\}$ crystal surface (T001) were synthesized by a modified HF hydrothermal method according to the previous report. ${ }^{21}$ In a typical procedure, $1.2 \mathrm{~mL}$ HF (aq) (0.6 mL 48\% wt HF mixed with $0.6 \mathrm{~mL}$ deionized water) were added dropwise into the $10 \mathrm{~mL}$ of tetrabutyl titanate solution under stirring for $1 \mathrm{~h}$. Then, the suspension was transferred into a $50 \mathrm{~mL}$ Teflon-lined autoclave and kept at $200{ }^{\circ} \mathrm{C}$ for $24 \mathrm{~h}$. The precipitate was collected and washed with water and ethanol. After drying, the powder was dispersed in $0.1 \mathrm{M} \mathrm{NaOH}$ (aq), stirred for $3 \mathrm{~h}$ to remove the adsorbed fluorine ions, and then washed with deionized water until neutral $(\mathrm{pH} \approx 7)$.

\section{Sample characterization}

Scanning electron microscopy (SEM, Hitachi SU8230, operated at $1 \mathrm{kV}$ acceleration voltage) and X-ray diffraction (XRD, Bruker 
D8 Discover, $\mathrm{Cu} \mathrm{K}_{\alpha 1}$, Bragg-Brentano) were used to analyze the morphology and phase purity of the as-synthesized $\mathrm{TiO}_{2}$ nanoparticles. Transmission electron microscopy (TEM) and highresolution TEM (HRTEM) were performed on an FEI Tecnai$\mathrm{G}^{2} \mathrm{~F} 30$ transmission electron microscope at $200 \mathrm{kV}$ to determine the lattice parameters of the three samples. The surface adsorbates were detected using in situ FTIR (Bruker Vertex 70) equipped with a high-temperature and high-pressure transmission IR-cell (Harrick Scientific Products Inc, NY, US) and a mercury cadmium telluride (MCT) detector. Specifically, the samples were outgassed under vacuum $\left(p<3 \times 10^{-3} \mathrm{mbar}\right)$. In situ measurements under flowing $\mathrm{O}_{2}\left(15 \mathrm{~mL} \mathrm{~min}{ }^{-1}\right)$ were performed during ramps from 30 to $400{ }^{\circ} \mathrm{C}$ at $5{ }^{\circ} \mathrm{C} \mathrm{min}^{-1}$. The applied water pressure was fixed with $0.35 \mathrm{RH}$ at $30^{\circ} \mathrm{C}$. BET surface analyses were operated with nitrogen physisorption measurements in the range of pressure of $0.6587 \mathrm{kPa}$ to 101.48 $\mathrm{kPa}$ at liquid nitrogen temperature.

\section{Conductivity measurements}

The as-prepared samples (T101, T001, and T100) were compacted into pellets with a diameter of $13 \mathrm{~mm}$ using a uniaxial cold press and then sintered at $400{ }^{\circ} \mathrm{C}$ for $4 \mathrm{~h}$ in ambient air. The thickness was about $1.5 \mathrm{~mm}$, and the relative density was about $50 \%$ calculated from the weight and geometry of the pellet. $\mathrm{Ag}$ ink was painted as electrodes on both sides of the pellet that was then dried at $120{ }^{\circ} \mathrm{C}$ for $20 \mathrm{~min}$. All the electrical measurements were carried out in a ProboStat ${ }^{\mathrm{TM}}$ sample holder cell (NORECS, Norway) with a standard 2-electrode 4-wire set up connected to a Novocontrol alpha-A impedance spectrometer via a ZG4 interface. A steel outer tube was used to electrically shield the cell. The impedance spectra were recorded from $10 \mathrm{MHz}$ to 1 $\mathrm{mHz}$ at oscillation voltages of $0.1-0.3 \mathrm{~V}$ RMS in $\operatorname{dry}$ or wet $\left(P_{\mathrm{H}_{2} \mathrm{O}}=\right.$ 0.026 atm or $\left.P_{\mathrm{D}_{2} \mathrm{O}}=0.023 \mathrm{~atm}\right)$ air as a function of temperature $\left(25-400{ }^{\circ} \mathrm{C}\right)$. The data were analyzed and modelled using the ZView equivalent circuit fitting software (ZView ver. 3.5a; Scribner Associates Inc.). The specific conductivity, $\sigma$, of the porous material was calculated from the macroscopic dimensions, i.e., the area of the electrodes and thickness of the pellet.

\section{Results and discussion}

\section{Structure characterization}

To investigate the morphology and crystal structure of the synthesized $\mathrm{TiO}_{2}$ samples, XRD, SEM, HRTEM, and BET characterizations were performed. Fig. 1 shows the XRD patterns, matching well with the anatase phase (tetragonal, JCPDS 211272). Obviously, the (004) diffraction line of the T001 sample weakened and its full width at half maximum (FWHM) broadened. According to the Scherrer equation, the broad peak represents the decreased crystallite size along the [001] axis vertical to (001) plane, corresponding to a smaller thickness in that direction. ${ }^{22}$ In addition, the (200) peak intensified and its FWHM narrowed, revealing the increased side length of T001 nanosheets in the [100] direction. Therefore, the thickness and side length in the [001] and [100] direction, respectively, can be roughly estimated based these two peaks. The relative peak

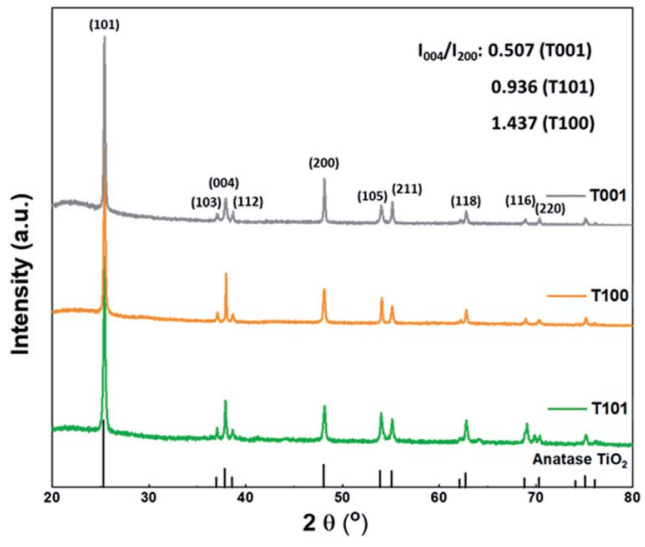

Fig. 1 XRD patterns of the as prepared $\mathrm{TiO}_{2}$ samples.

intensity of (004) and (200) was estimated in the insert. T001 has the lowest value, indicative of dominance of $\{001\}$ facets. ${ }^{23}$ For the T100 sample, changes of the (004) and (200) peaks show an opposite trend, suggesting that the crystals grew preferentially in the [001] direction, while the growth in the [100] direction was suppressed. ${ }^{24}$.

Fig. 2a shows the rectangular sheet-like shape of the T001 sample. The HRTEM image and the corresponding fast Fourier transform (FFT) displayed in the inset of Fig. 2a clearly show the lattice spacing of around $0.189 \mathrm{~nm}$, which corresponds to the (001) plane of anatase. Fig. 2b suggests that most of the T100 particles have a tetragonal faceted nanorod morphology. Three sets of clear lattice fringes with spacing of $0.35 \mathrm{~nm}, 0.35 \mathrm{~nm}$, and $0.48 \mathrm{~nm}$, respectively, can be attributed to the (101), (101) and (002) planes of the anatase phase, which is typical for the $\{100\}$ facet. ${ }^{25}$ The images of the T101 sample (Fig. 2c) show the truncated bipyramidal shape with the bottom and top surfaces being composed of the $\{001\}$ facet and either side surfaces found to be the $\{101\}$ facet based on previous literature findings. The lattice fringe with a d-spacing of $0.35 \mathrm{~nm}$ corresponds to (101) planes of anatase. ${ }^{26}$ Additional SEM images and indicative particle dimensions of each sample can be found in Fig. S1. $\dagger$ Specific surface areas as calculated from $\mathrm{N}_{2}$ adsorption isotherms at $77 \mathrm{~K}$ (Table $\mathrm{S} 1 \dagger$ ) are higher for the T001 sample than the other two, attributed to its thin sheet-like morphology.

\section{$\mathrm{H}_{2} \mathrm{O}-\mathrm{TiO}_{2}$ interactions}

The surface of $\mathrm{TiO}_{2}$ has both Lewis basic $\left(\mathrm{O}_{2 \mathrm{c}}\right)$ and acidic $\left(\mathrm{Ti}_{5 \mathrm{c}}\right)$ sites. Water adsorbs molecularly, which may be followed by dissociation, ${ }^{\mathbf{1 2}}$ as illustrated in Fig. 3. Molecular adsorption involves a direct interaction of the oxygen atom of $\mathrm{H}_{2} \mathrm{O}\left(\mathrm{O}_{\mathrm{w}}\right)$ with the surface $\mathrm{Ti}^{4+}$ acidic site $\left(\mathrm{Ti}_{5 \mathrm{c}}\right)$, or/and the hydrogen atom of $\mathrm{H}_{2} \mathrm{O}\left(\mathrm{H}_{\mathrm{w}}\right)$ with the surface $\mathrm{O}^{2-}$. In the later dissociation, a basic site $\left(\mathrm{O}_{2 \mathrm{c}}\right)$ can extract a proton from the water molecule that was adsorbed on the adjacent $\mathrm{Ti}_{5 \mathrm{c}}$, forming two surface hydroxyl groups (the terminal hydroxyl group, $\mathrm{OH}_{\mathrm{t}}$, and the bridging hydroxyl group, $\left.\mathrm{OH}_{\mathrm{b}}\right){ }^{27}$

As discussed above, the surface atom coordination and geometric arrangement, as well as the resulting steric 

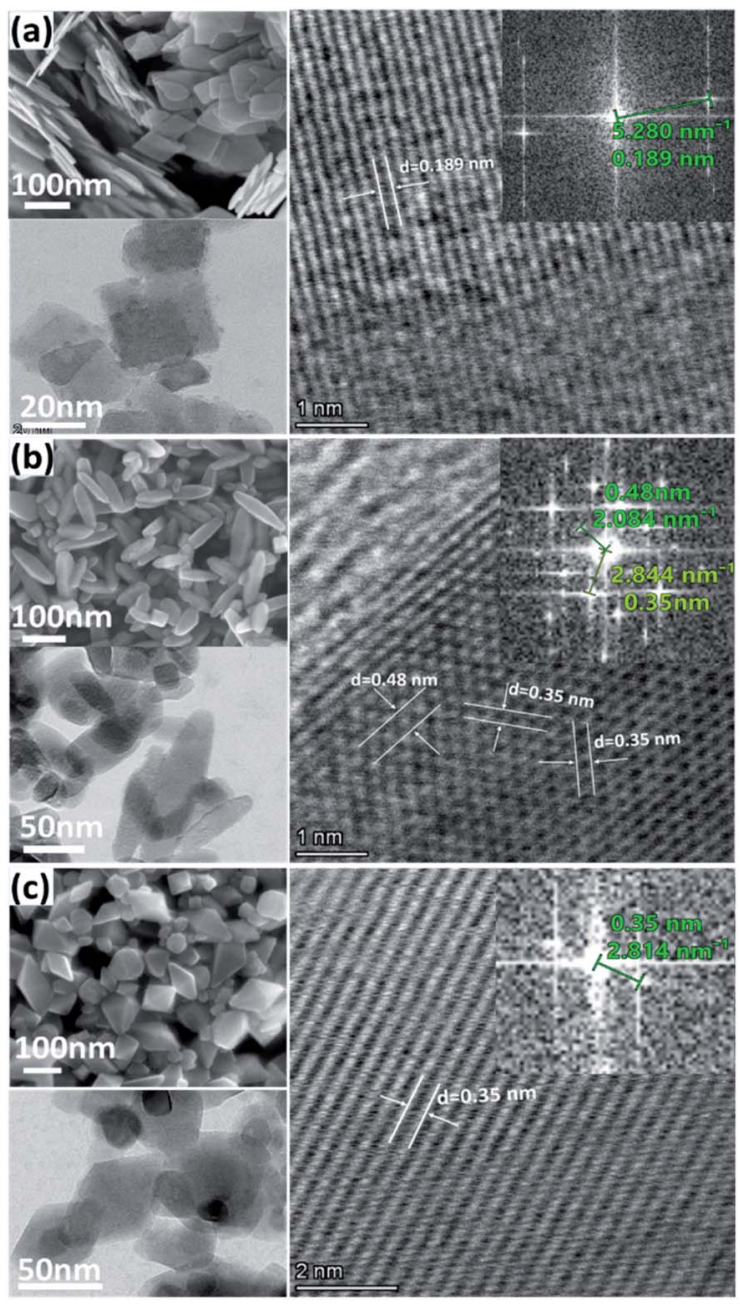

Fig. 2 Typical SEM, TEM, and HRTEM images and the corresponding FFT patterns of (a) T001, (b) T100, and (c) T101 sample.

hindrance considerably affect the behavior on water adsorption on different anatase facets. However, previous studies investigating water adsorption and desorption were mainly conducted at very low temperatures and ultra-high vacuum (UHV), ${ }^{12}$ where $p_{\mathrm{H}_{2} \mathrm{O}}$ is low, but $\mathrm{RH}$ is considerable. In practical applications at ambient temperatures, $p_{\mathrm{H}_{2} \mathrm{O}}$ is higher, and $\mathrm{RH}$ is still high. At higher temperatures, $\mathrm{RH}$ is often lower, resulting in a lower coverage of adsorbed water. A main effect of increased temperature, even at the same $\mathrm{RH}$, is increased dissociation of adsorbed molecular water. The question is how the thermodynamics of adsorption and dissociation vary with the configuration of surface sites on different facets.

Fig. 4 shows the FTIR spectra of the as-prepared $\mathrm{TiO}_{2}$ samples outgassed at elevated temperatures. From the evolution of $-\mathrm{OH}$ absorbance with temperatures, the degree of dehydration and thermal stability of various $-\mathrm{OH}$ groups is indicated. We can distinguish three main IR absorption bands. A broad absorption band at around $3600-2800 \mathrm{~cm}^{-1}$ corresponds to the stretching vibration modes of $-\mathrm{OH}$ group, $\nu(\mathrm{OH})$, and the $\mathrm{H}_{2} \mathrm{O}$ molecule perturbed by hydrogen bonding (Fig. 4a). The broadness is related to the length and strength of several possible $\mathrm{O}-\mathrm{H}$ bonds in a dynamic intermolecular interaction. A series of sharp peaks at around $3600-3750 \mathrm{~cm}^{-1}$ correspond to the unperturbed terminal hydroxyls and water molecules. ${ }^{28}$ Peaks below $1800 \mathrm{~cm}^{-1}$ can be assigned to the bond bending of $-\mathrm{OH}$ groups, $\delta(\mathrm{OH})$. Clearly, the amount of adsorbed water at the same RH is more significant on the $\{001\}$ and $\{100\}$ facets, probably due to their higher surface energy with a large amount of under-coordinated sites compared to that of the $\{101\}$ facet.

The evolution of the FTIR spectra due to the changed surface hydration species by the progressive outgassing from $30{ }^{\circ} \mathrm{C}$ to $400{ }^{\circ} \mathrm{C}$, was monitored. As shown in Fig. $4 \mathrm{~b}-\mathrm{d}$, the integrated intensity in $3600-2800 \mathrm{~cm}^{-1}$ gradually decreases with increasing temperature, and is completely flattened out at $400{ }^{\circ} \mathrm{C}$ for all the three $\mathrm{TiO}_{2}$ samples, reflecting the condensation of $-\mathrm{OH}$ groups and desorption of water. ${ }^{28}$ The change in peak intensities on the T101 and T001 surfaces as the temperature increased from 30 to $40{ }^{\circ} \mathrm{C}$ indicates the massive loss of water on these two surfaces.

Previous studies suggested that the formation of the ice-like layer is almost completed at $0.3 \mathrm{RH}$, and at $0.3-0.6 \mathrm{RH}$, the second physisorbed water layer forms. ${ }^{29}$ Here, we propose that the chemisorbed water layer dissociates and mainly consists of $-\mathrm{OH}_{\mathrm{t}}$ and $-\mathrm{OH}_{\mathrm{b}}$ groups on the $\{001\}$ and $\{100\}$ facets, while it remains mainly undissociated $\mathrm{H}_{2} \mathrm{O}$ molecules $\left(\mathrm{Ti}_{5 \mathrm{c}}-\mathrm{H}_{2} \mathrm{O}\right)$ on the $\{101\}$ facet. In the first physisorbed water layer, the incoming $\mathrm{H}_{2} \mathrm{O}$ forms double or single hydrogen bonds with the hydroxyls underneath on the surface of T001 and T100, making this layer rigid enough to be ice-like. Since the density of $-\mathrm{OH}_{\mathrm{b}}$ on $\{001\}$ is the highest (100\% O-2c sites) among these three facets, more $\mathrm{OH}_{\mathrm{b}}-\mathrm{H}_{2} \mathrm{O}$ hydrogen bonds are expected. On the $\{101\}$ surface, however, instead of forming hydrogen bonds with $\mathrm{Ti}_{5 \mathrm{c}}-\mathrm{H}_{2} \mathrm{O}$, the incoming $\mathrm{H}_{2} \mathrm{O}$ adsorbs molecularly on surface $\mathrm{O}_{2 \mathrm{c}}$ sites due to the unique zigzag configuration of the $\{101\}$ facet. $^{30,31}$ The aforementioned ice-like physisorbed water layer follows after the formation of the $\mathrm{O}_{2 \mathrm{c}}-\mathrm{H}_{2} \mathrm{O}$ is completed and therefore at
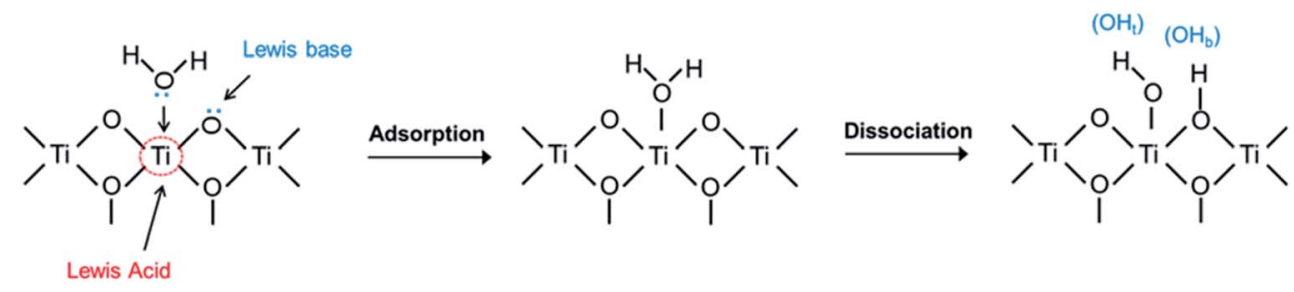

Fig. 3 Schematic illustration of adsorption and dissociation of water on $\mathrm{TiO}_{2}$ surfaces. 

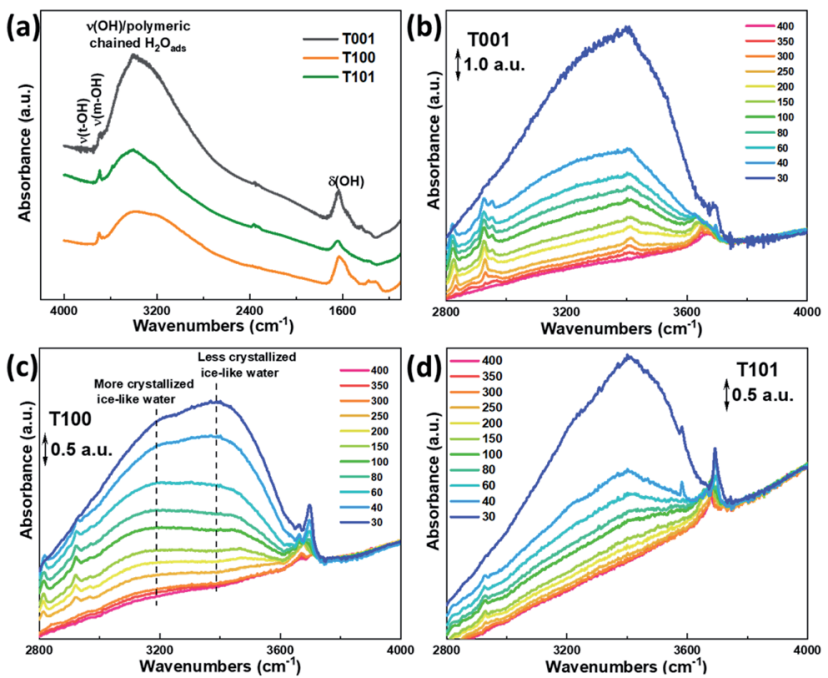

Fig. 4 (a) FTIR spectra showing the water adsorption behaviors on the different $\mathrm{TiO}_{2}$ samples at $30{ }^{\circ} \mathrm{C}$, and in situ FTIR spectra of water adsorption on (b) T001, (c) T100 and (d) T101 samples at different temperatures (in ${ }^{\circ} \mathrm{C}$ ) with progressive water vapor removal. The scale bar of the arbitrary units (a.u.) of the $y$ axis are provided in $(b-d)$ for comparison.

higher RH compared to the ice-like layer on $\{001\}$ and $\{100\}$ facets.

Above $0.3 \mathrm{RH}$, the second physisorbed water layer develops by weaker hydrogen bonds, and the outermost layer changes gradually from ice-like to liquid-like. ${ }^{29}$ During the degassing process, a massive water loss at $40{ }^{\circ} \mathrm{C}$ is observed for the $\{001\}$ (Fig. 4b), reflecting the weak hydrogen bonds in the outmost water layer. Therefore, it appears that the second physisorbed water layer that is thermally unstable forms on the $\{001\}$ surface at $30{ }^{\circ} \mathrm{C}$ with $0.35 \mathrm{RH}$, while its formation is less favorable on the $\{101\}$ and $\{100\}$ under these conditions.

As for the T101 sample, most water molecules desorb at temperatures up to $150{ }^{\circ} \mathrm{C}$, whereas they are still visible on the $\{001\}$ and $\{100\}$ facets at around $200-250{ }^{\circ} \mathrm{C}$ (Fig. $4 \mathrm{~b}-\mathrm{d}$ ), indicating the unstable nature of the less crystallized ice-like layer on $\{101\}$ surface due to the lack of hydroxyls underneath, as well as the relatively weak interaction of water hydrogen and surface oxygen. Noticeably, a lower-lying absorbance maximum at around $3200 \mathrm{~cm}^{-1}$ is more visible for the T100 than the other two samples (Fig. 4c), which can be ascribed to the more crystalline (ice-like) water. In comparison, the one at around $3400 \mathrm{~cm}^{-1}$ is from the more amorphous and liquid-like water. ${ }^{32}$ Hence, it is reasonable to infer that a crystallized ice-like water layer is established on the surface of these three samples during the in situ FTIR test, but the ice-like layer is more crystallized on the $\{100\}$ surface than the other two.

A detailed analysis at the $3600-3750 \mathrm{~cm}^{-1}$ region where peaks are persistent even at $400{ }^{\circ} \mathrm{C}$ is displayed in Fig. 5. For the T001 sample, two peaks at around $3651 \mathrm{~cm}^{-1}$ and $3677 \mathrm{~cm}^{-1}$ can be ascribed to $\mathrm{OH}_{\mathrm{b}}$ and $\mathrm{OH}_{\mathrm{t}}$, respectively. When increasing the temperature to $400{ }^{\circ} \mathrm{C}$, they positively shift to $3660 \mathrm{~cm}^{-1}$ and $3683 \mathrm{~cm}^{-1}$, respectively, due to the disappearance of the additional water molecules bonding to these $-\mathrm{OH}$ species. ${ }^{33,34} \mathrm{~A}$ peak at around $3695 \mathrm{~cm}^{-1}$ is only seen in the low-temperature range (below $100{ }^{\circ} \mathrm{C}$ ), which can be assigned to the free $\mathrm{H}_{2} \mathrm{O}$ in the second physisorbed layer where the outermost layer with less $\mathrm{H}-$ bonds is involved. A similar change takes place on the T100 sample, in which the two peaks at $3663 \mathrm{~cm}^{-1}\left(\mathrm{OH}_{\mathrm{b}}\right)$ and $3697 \mathrm{~cm}^{-1}$ $\left(\mathrm{OH}_{\mathrm{t}}\right)$ shift to $3670 \mathrm{~cm}^{-1}$ and $3715 \mathrm{~cm}^{-1}$, respectively, as temperatures increase to $400{ }^{\circ} \mathrm{C}$. Noticeably, compared to T001, the $\mathrm{OH}_{\mathrm{t}}$ signal is more pronounced than $\mathrm{OH}_{\mathrm{b}}$ on T100 surfaces, which is probably due to its higher density of $\mathrm{Ti}_{5 \mathrm{c}}(100 \%)$ than $\mathrm{O}_{2 \mathrm{c}}(50 \%)$.

With regard to the T101 sample (Fig. 5), only one distinctive peak appears, which positively shifts from $3691 \mathrm{~cm}^{-1}$ to $3695 \mathrm{~cm}^{-1}$ with the temperature increasing from 30 to $150{ }^{\circ} \mathrm{C}$, then negatively shifts to $3670 \mathrm{~cm}^{-1}$ at $400{ }^{\circ} \mathrm{C}$. Based on the above discussion, this single peak belongs to $\mathrm{H}_{2} \mathrm{O}$ molecularly adsorbed on the $\{101\}$ surface, and the peak-shifting probably originates from the desorption of water from $\mathrm{Ti}_{5 \mathrm{c}}-\mathrm{H}_{2} \mathrm{O}, \mathrm{O}_{2 c^{-}}$ $\mathrm{H}_{2} \mathrm{O}$, and $\mathrm{H}_{2} \mathrm{O}-\mathrm{H}_{2} \mathrm{O}$ species as temperature increases. The above explanation is further supported by a detailed analysis of peak evolution in $\mathrm{O}-\mathrm{H}$ bending region in conjunction with Fig. S2. $\dagger$ It is worth noting though that calculations by Tilocca and Seloni indicated that molecular adsorption is favored in the case of a defect-free $\{101\}$ surface, while a defective surface containing oxygen vacancies can induce dissociative adsorption, attributed to $\mathrm{H}_{2} \mathrm{O}-\mathrm{H}_{2} \mathrm{O}$ interactions. ${ }^{31}$ Further investigations are needed to elucidate the degree of dissociative adsorption in relation to the amount of oxygen vacancies in the surface.
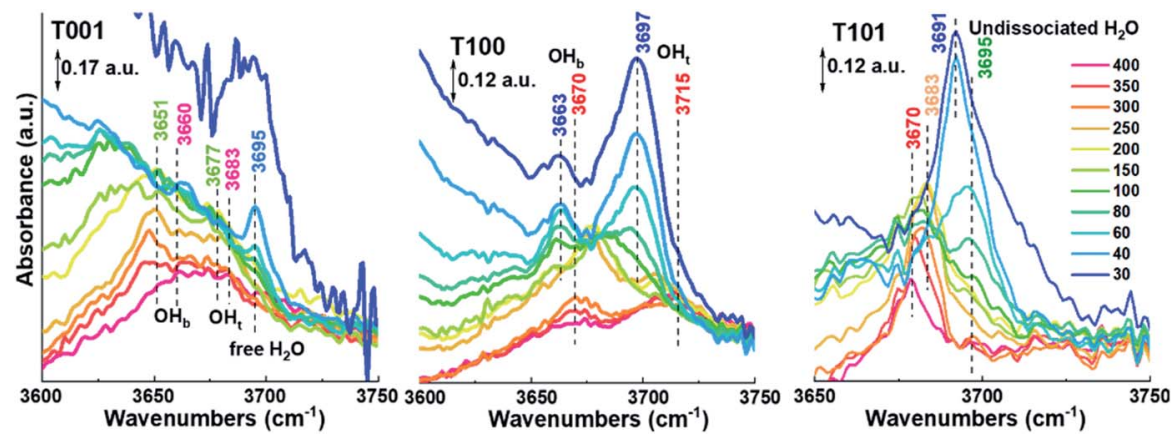

Fig. 5 FTIR spectra in the high wavenumber range of $\mathrm{TiO}_{2}$ samples presented for different temperatures (in ${ }^{\circ} \mathrm{C}$ ) and progressive water vapor removal. The colors of the peak numbers correspond to the different temperatures. 
Based on the above discussion, water layers adsorbed on different facets of $\mathrm{TiO}_{2}$ are displayed in Fig. 6. As the $\mathrm{RH}$ increases, different water layers gradually form on the surface of these three $\mathrm{TiO}_{2}$ samples. Here, we use the $\{001\}$ facet as an example to show all these water layers. The first chemisorbed water layer forms at temperatures above $275{ }^{\circ} \mathrm{C}$ with mainly hydroxyls $\left(\mathrm{OH}_{\mathrm{b}}\right.$ and $\left.\mathrm{OH}_{\mathrm{t}}\right)$ on $\{001\}$ and $\{100\}$ facets and mainly molecularly adsorbed water on $\{101\}$ facets. These results are in line with several theoretical findings in the literature..$^{35-37}$

As RH increases, a hierarchical ice-like water layer starts forming on the top of the chemisorbed layer on $\{001\}$ and $\{100\}$ facets. On the $\{101\}$ surface, the incoming water molecules first bond to $\mathrm{O}_{2 \mathrm{c}}{ }^{30}$ and then to the surface adsorbed $\mathrm{H}_{2} \mathrm{O}$ with hydrogen bonds are not as strong as those on the $\{001\}$ and $\{100\}$ facets where water molecules form hydrogen bonds with the surface $-\mathrm{OH}$ groups. The ice-like water layer is more rigid on the $\{100\}$ facet than that on the $\{001\}$ because of its special surface atomic arrangement. Previous theoretical calculations indicated that the coinciding orientations of water adsorbed on the surface active sites of $\{100\}$ facets (with respect to the $\mathrm{TiO}_{2}$ surface plane) yield hydration layers with an enhanced density, and lead to strongly ordered water layers. ${ }^{\mathbf{1 6}}$ As the $\mathrm{RH}$ further increases, a third water layer physisorbs first on $\{001\}$ surfaces, and propagates further into liquid-like layers at RH above 0.6.

As discussed in the introduction, the formation of protonic charge carriers originates from the surface water-oxide interactions, while the conduction of these charge carriers takes place in all water layers. Therefore, the proton conduction mechanism and conductivity on different crystal facets are expected to be different.

\section{Conductivity measurements}

Impedance spectra were measured for all samples as a function of temperature in various atmospheres. Temperatures were kept below $400{ }^{\circ} \mathrm{C}$ to avoid sintering and change of crystal

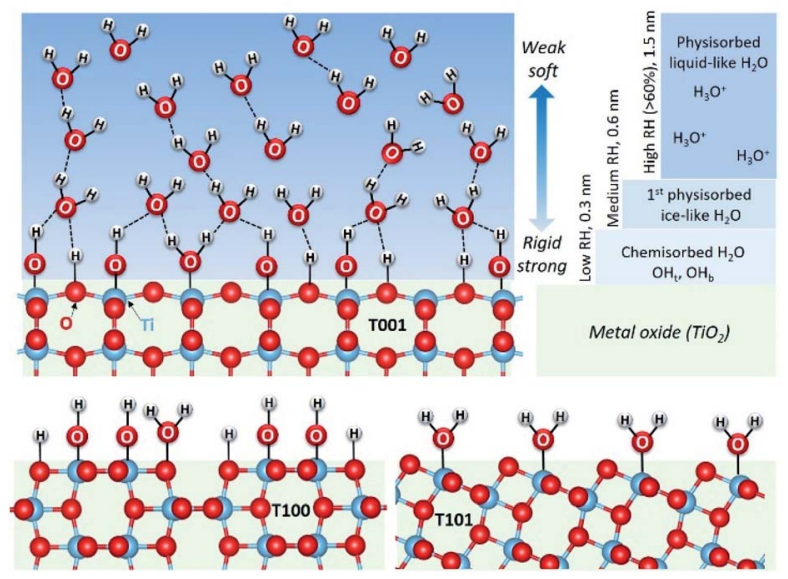

Fig. 6 Schematic presentation of the proposed structures of water layers adsorbed on the studied facets of $\mathrm{TiO}_{2}$. Main and different characteristics of the first, chemisorbed layer are shown on all, while the more common features of physisorbed layers are shown only for $\{001\}$. facets. A representative Nyquist plot of the impedance at $50{ }^{\circ} \mathrm{C}$ of the $\{101\}$ facet dominated $\mathrm{TiO}_{2}$ sample, and the corresponding simplified equivalent circuit used to model all spectra, are displayed in Fig. S3. $\dagger$ Based on the associated capacitances, the impedance at high frequencies is ascribed to the transport in the bulk and over the surface of the grains. Although the high frequency impedance arc is asymmetrical, our data don't allow a consistent further deconvolution of this impedance, and we here assign only one resistance and conductivity to it. The response at low frequencies is assigned to the electrode impedance. The inset in Fig. S3† shows the impedance in nominally dry air that is orders of magnitude higher at this temperature.

Based on impedance spectra and sample geometries, the conductivities of the different samples under both dry and wet conditions were extracted and form basis for Fig. 7. The curves were fitted with TableCurve 2D software. The pre-exponentials $\left(\sigma_{0}\right)$ and activation energies $\left(E_{\mathrm{a}}\right)$ of total conductivities corresponding to the bulk transport, the chemisorbed water layer, and the ice-like physisorbed layer were obtained separately, as shown in Fig. 7a, and the numerical results are provided in Table S2. $\dagger$ For the T101 sample, we fitted the $\sigma_{0}$ and $E_{\mathrm{a}}$ for four different contributions: bulk transport, and transport in the $\mathrm{Ti}_{5 \mathrm{c}}-\mathrm{H}_{2} \mathrm{O}$ layer, $\mathrm{O}_{2 \mathrm{c}}-\mathrm{H}_{2} \mathrm{O}$ layer, and ice-like physisorbed layer.

For nominally dry conditions, the data, shown only at the highest temperatures in Fig. 7c-e (region I), exhibit linear Arrhenius behaviors with activation enthalpies $\left(E_{\mathrm{a}}\right)$ calculated from $\log (\sigma T)$ vs. $1 / T$ in the range of $0.8-1.2 \mathrm{eV}$. This can be attributed to the ionic conductivity of nanometric anatase, ${ }^{38,39}$ and the variation between samples may be understood by their
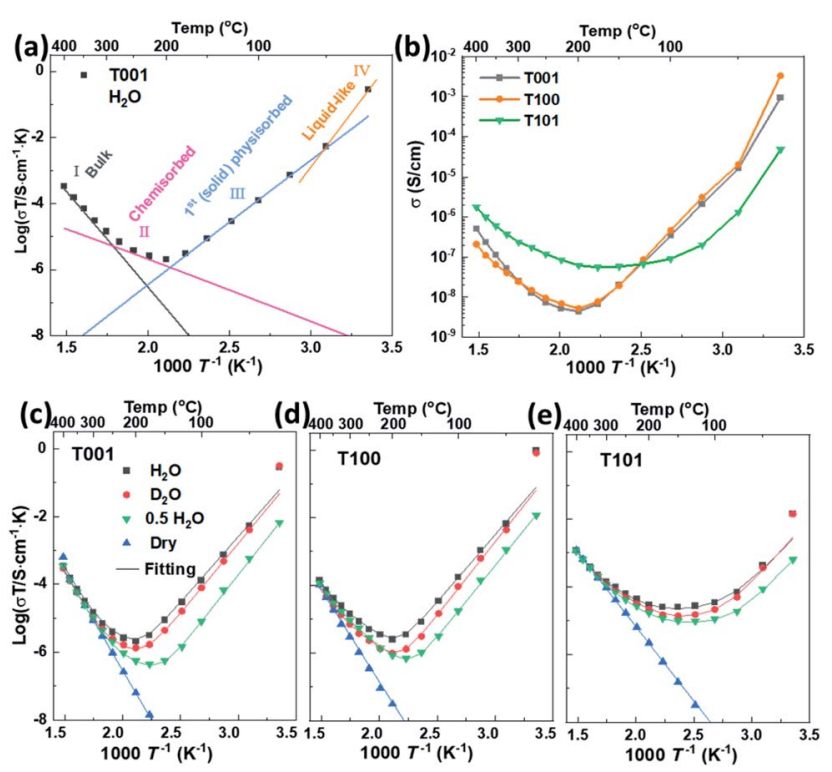

Fig. 7 (a) Schematic illustration of the fitted conductivity at different temperatures with $\mathrm{T001}$ as example. (b) Total conductivity of $\mathrm{TiO}_{2}$ samples with predominance of different crystal facets extracted from impedance spectra under wet air $\left(p_{\mathrm{H}_{2} \mathrm{O}}=0.026 \mathrm{~atm}\right)$. The total conductivity as $\log (\sigma T)$ of (c) T001, (d) T100 and (e) T101 extracted from impedance spectra in dry and wet air $\left(p_{\mathrm{H}_{2} \mathrm{O}}=0.026 \mathrm{~atm}, p_{\mathrm{D}_{2} \mathrm{O}}=0.023\right.$ atm, $p_{\mathrm{H}_{2} \mathrm{O}}=0.013 \mathrm{~atm}\left(" 0.5 \mathrm{H}_{2} \mathrm{O}\right.$ ")) vs. $1 / T$. 
different textures and dominance of different crystallographic directions. Under wet conditions, the conductivity at the highest temperatures is still dominated by bulk transport and does not deviate much from that under dry conditions.

Below approximately $300{ }^{\circ} \mathrm{C}$, the surface protonic contribution becomes significant under wet conditions, giving rise to activation enthalpies in region II (chemisorbed water layer) in the range $0.3-0.4$ for T001, $0.4-0.5 \mathrm{eV}$ for T100 and $0.4-0.6 \mathrm{eV}$ for T101. The difference between samples is clear from the direct comparison of the conductivities in wet atmosphere $\left(p_{\mathrm{H}_{2} \mathrm{O}}\right.$ $=0.026 \mathrm{~atm}$ ) in Fig. $7 \mathrm{~b}$. The conductivity in range II is attributed to mainly chemisorbed water, with proton migration by the Grotthuss mechanism. ${ }^{3}$ For T001 and T100 we expect that the chemisorbed layer is at full coverage and fully dissociated, so that the activation energies reflect the enthalpy of proton charge mobility (diffusion). They are in overall agreement with the $58 \mathrm{~kJ} \mathrm{~mol}^{-1}(0.60 \mathrm{eV})$ found for bulk proton migration in polycrystalline rutile and within the range of proton diffusivities in the different directions of single crystal rutile ${ }^{40}$ knowing that surface transport has somewhat lower activation enthalpies than in bulk. The activation energy of T101 should, on the one hand, contain some dissociation enthalpy, but may on the other hand reflect easier jumps between the loosely bonded water molecules on the surface of T101 than the more rigid and dissociated water on T001 and T100.

At temperatures below approximately $200{ }^{\circ} \mathrm{C}$ (region III, $1^{\text {st }}$ physisorbed layer), the conductivity increased with decreasing temperature for T001 and T100, as expected, attributed to the protolysis of the dissociated protons from the chemisorbed layer into the increasing coverage of physisorbed water, where the mobility is higher. ${ }^{41,42}$ The conductivities down to around $50{ }^{\circ} \mathrm{C}$ follow remarkably straight lines in the Arrhenius plot corresponding to apparent enthalpies of around $-0.8 \mathrm{eV}$. For T101, we get instead a shallow conductivity minimum in region III, suggested to originate from the continued adsorption of $\mathrm{H}_{2} \mathrm{O}$ on $\mathrm{O}_{2 \mathrm{c}}$ sites, and the sharper increase due to the more continuous molecular physisorbed layer appears only around $100{ }^{\circ} \mathrm{C}$. Whether this is ice-like on T101 like on T001 and T100 is uncertain, but it still comes with the high mobility.

The observed negative activation energy for the temperature dependency of proton conductivity in the ice-like physisorbed water in range III have several contributions: the enthalpy of adsorption of the first physisorbed layer should correspond to something a bit more negative than that of condensation of water $(-0.45 \mathrm{eV}$ at RT). But the enthalpy of mobility goes in the opposite direction. To get to $-0.8 \mathrm{eV}$, we must therefore expect that the increasing coverage of the first physisorbed layer increases the degree of protolysis ${ }^{31}$ and decreases the activation enthalpy of mobility. What appears as a negative temperature dependence with an enthalpy of $-0.8 \mathrm{eV}$ is actually a continuously decreasing (yet positive) activation enthalpy of conduction with increasing coverage, as pointed out by others before. ${ }^{43}$ One may say that the enthalpy of conduction is positive, but the differential enthalpy is negative.

The contribution from entropy with respect to the formation and conduction of protons should also not be neglected in rationalizing the apparent negative enthalpy. As the first physisorbed water layer gains in coverage, protons can move into it at lower density (concentration), which increases both the vibrational and configurational entropy and drives the water protolysis forward, and, in turn, dissociation to increase the total number of mobile protons.

At temperatures below $50{ }^{\circ} \mathrm{C}$ (in ambient humidity, with $p_{\mathrm{H}_{2} \mathrm{O}}$ $=0.026 \mathrm{~atm}$ ), region IV, the conductivities of T001 and T100 increase even more sharply with decreasing temperature, reflecting increasingly high vehicular mobility of protons in the thicker and liquid-like physisorbed layers. The mobility is approaching that of bulk water, independent of the characteristics of the oxide surface. Thus, the different conductivities observed on these three facets are now mostly determined by the concentration of protonic species.

Sample T101, which displayed a lower activation energy of proton conductivity in the chemisorbed layer and hence had a higher conductivity than the others at the minima around $150{ }^{\circ} \mathrm{C}$, does not exhibit the behavior attributed to the first physisorbed layer that we saw in T001 and T100. Instead, it appears that it goes more directly from conduction in chemisorbed water to liquid-like physisorbed water layers, in agreement with the conclusions from the FTIR studies. It should be noted that the apparent activation energy of T101 is less negative than that of T001 and T100 at temperatures from 150 to $50{ }^{\circ} \mathrm{C}$, during which the first physisorbed water layer forms. For $\{001\}$ and $\{100\}$ facets, water molecules form hydrogen bonds with surface $-\mathrm{OH}$ groups, while they are bonded to the molecular water on $\{101\}$ facets in the chemisorbed water layer, which decreases the magnitude of the enthalpy of the water adsorption/condensation process (makes it less exothermic). The increasing coverage of the first physisorbed layer with decreasing temperature hence lags behind for T101 as compared to T001 and T100, the apparent enthalpy gets less negative, and the conductivity increases less sharply.

T101 ends up at 1-2 orders of magnitude lower conductivity than T001 and T100 at room temperature with a $\mathrm{RH}$ of $80 \%$. Assuming that this reflects the concentration of protons, this must, in turn, stem from a lower degree of dissociation and/or protolysis. A lower degree of dissociation is in agreement with what FTIR analyses concluded about molecular water adsorption on the $\{101\}$ facet. Another possibility lies in lower adsorption energies of water on the $\{101\}$ facet resulting in lower total thickness and less liquid-like character of water here.

Interestingly, the pre-exponential factors acquired from the fitting of conductivities in the chemisorbed water are higher for T001 and T100 than for T101, see Table S2. $\dagger$ This reflects only differences in entropy, attempt frequencies, and density of sites. We suggest that it reflects that the $\{101\}$ facet has much fewer active sites for adsorption and dissociation of water.

All in all, we find that surfaces with dissociative chemisorption exhibit high surface protonic conductivity at the highest and lowest temperatures, due to a high concentration of sites for adsorption and dissociation and unrestricted mobilities for two very different reasons. At intermediate temperatures, however, they exhibit deep minima from the high activation energies for mobility in the chemisorbed and the 
rigid first physisorbed layers. Surfaces with less dissociation and hence molecular chemisorption, on the other hand, appear to offer fewer protons (fewer sites for adsorption and dissociation) but cross the temperature range with a much shallower minimum at intermediate temperatures because the activation energies are smaller.

The findings and conclusions above were supported by H/D isotope effects. The classical model predicts that the proton hopping rate in oxides is proportional to the $\mathrm{O}-\mathrm{H} / \mathrm{O}-\mathrm{D}$ vibration frequency, but the model has uncertainties related to sticking probability and other mechanistic aspects. Instead, the isotope effect stems mostly from a higher activation energy of jumps from $\mathrm{O}-\mathrm{D}$ vibrations due to different semiclassical zero-point energies of $\mathrm{O}-\mathrm{H}$ and $\mathrm{O}-\mathrm{D}$ compared to their $\mathrm{O}-\mathrm{H}-\mathrm{O}$ and $\mathrm{O}-\mathrm{D}-\mathrm{O}$ jump saddle point energies, accounting for observed $\mathrm{H}$ / D isotope effects. ${ }^{44}$ The significant isotope effect ratio of $\sim 2$ around $150{ }^{\circ} \mathrm{C}$ (Fig. 8) shows that Grotthuss free proton jumps is the dominating mechanism. It decreases with increasing temperatures as the effect of the different activation energies diminish $^{\mathbf{4 4}}$ and native bulk conductivity becomes significant. The isotope effect also gradually diminishes at temperatures below $150{ }^{\circ} \mathrm{C}$ (higher $\mathrm{RH}$ ) and almost disappears as RH surpassed $60 \%$, indicating a change to vehicular transport in liquid-like water layers.

The T001 sample was synthesized using HF as the capping agent. It is well-known that fluoride ions can bond strongly to $\mathrm{Ti}_{5 \mathrm{c}}$ sites and stabilize the surface, ${ }^{\mathbf{4 5}}$ therefore, fluoride ions would inevitably adsorb on the sample surface during synthesis. When the surface Ti atoms are terminated by fluoride ions, the strong bonding between the Ti-5c and water oxygen $\left(\mathrm{O}_{\mathrm{w}}-\mathrm{Ti}_{5 \mathrm{c}}\right)$ is no longer possible. Moreover, the hydrogen bond between the water hydrogen and surface $\mathrm{O}-2 \mathrm{c}\left(\mathrm{H}_{\mathrm{w}}-\mathrm{O}_{2 \mathrm{c}}\right)$ also becomes unfavorable due to the repulsive interactions between surface oxygen and fluoride ions. In this case, water layers cannot be formed on the surface of $\mathrm{TiO}_{2}$ at low temperatures, and as a result, the conductivity remained $<10^{-7} \mathrm{~S} \mathrm{~cm}^{-1}$ throughout the temperature range of interest (Fig. 9). However, when the surface absorbed fluoride ions

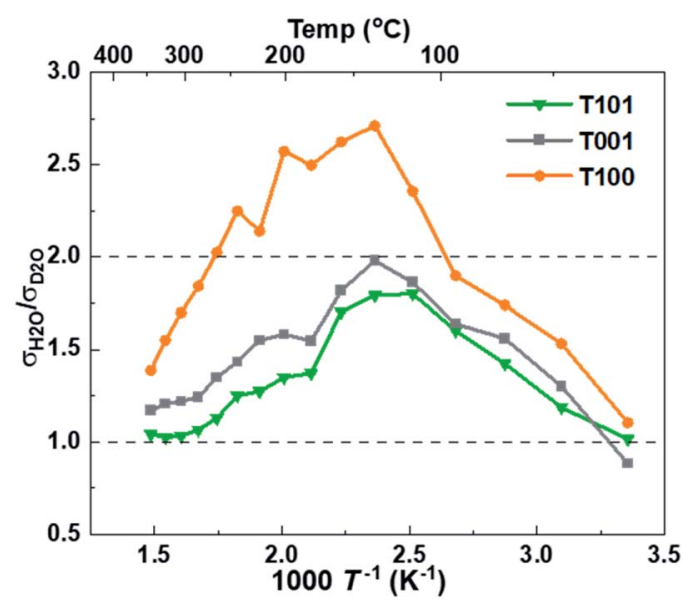

Fig. 8 The ratio of protonic over deuteronic conductivity in $\mathrm{H}_{2} \mathrm{O}$ and $\mathrm{D}_{2} \mathrm{O}$ atmospheres.

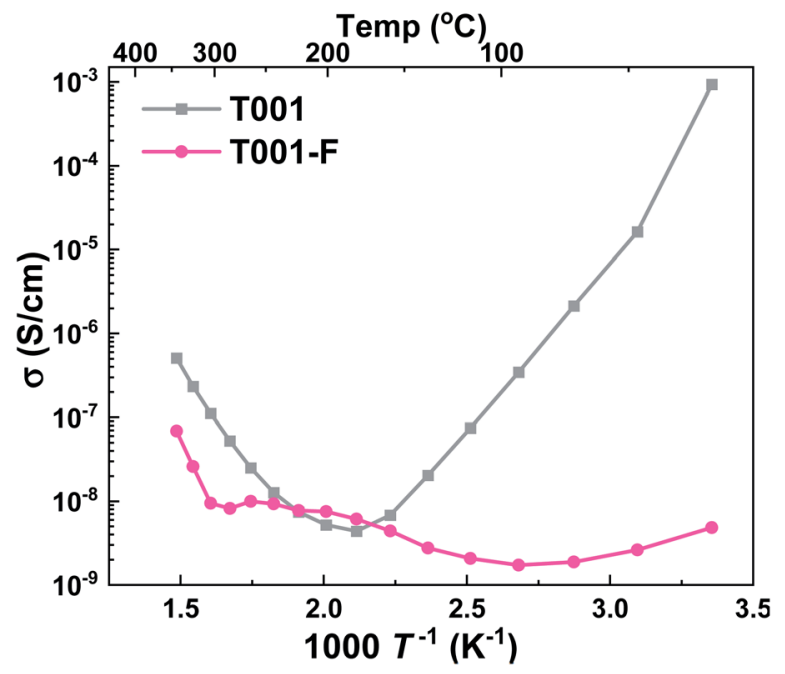

Fig. 9 Apparent conductivities in wet air of T001 samples capped with $\mathrm{HF}$ (T001-F) and after washing with $\mathrm{NaOH}$ solution to remove the surface adsorbed fluorine ions (T001).

were removed by washing with $\mathrm{NaOH}(\mathrm{aq}),{ }^{\mathbf{4 6 , 4 7}}$ the observed conductivity was greatly improved.

\section{Conclusions}

We systematically varied the dominance of different crystal facets and thereby surface chemistry of nanocrystalline $\mathrm{TiO}_{2}$ to shed light on their role in water-oxide interactions and proton conduction. In situ FTIR suggests that the coordination of surface atoms on $\{001\}$ and $\{100\}$ dominated facets makes water chemisorb strongly and dissociatively, while chemisorption on $\{101\}$ is weaker and more molecular. In ambient humidity $\left(p_{\mathrm{H}_{2} \mathrm{O}}\right.$ $=0.026 \mathrm{~atm}$ ) and below $300{ }^{\circ} \mathrm{C}$, surface protonic conduction in the chemisorbed layer becomes dominating, with activation enthalpies in the range of $0.4-0.6 \mathrm{eV}$ for $\{001\}$ and $\{100\}$ and $0.3-0.4 \mathrm{eV}$ for $\{101\}$. Then, the conductivity of all samples crosses a minimum, which is shallower for the $\{101\}$ surface because of the low activation energy for mobility in the soft molecular water. Below approximately $150{ }^{\circ} \mathrm{C}$, the coverage of the first ice-like physisorbed layer becomes significant, and its higher proton mobility increases the surface protonic conductivity with decreasing temperature. This sets in at higher temperatures and is steeper for $\{001\}$ and $\{100\}$ because physisorption is stronger (more exothermic) on the rigid dissociated than the soft molecular chemisorbed water underneath. Further down at $50{ }^{\circ} \mathrm{C}(\mathrm{RH} \approx 30 \%$ for ambient humidity) this layer is considered complete and as we approach room temperature and RH surpasses $60 \%$, liquid-like water layers physisorb, and the proton mobility and conductivity increase further. This is more pronounced on $\{001\}$ and $\{100\}$ due to more dissociation and thicker water layers, accommodating higher total concentrations of dissociated protons of higher mobility. This is supported by H/D isotope effect measurements showing a transition from Grotthuss $\mathrm{H}^{+}$hopping in the chemisorbed and first physisorbed layers to vehicular $\mathrm{H}_{3} \mathrm{O}^{+}$transport in the liquid-like outer layers. The surface chemistry was further 
studied by terminating $\{001\}$ facets with fluoride ions, which effectively suppressed surface protonic conduction by blocking $\mathrm{H}_{2} \mathrm{O}-\mathrm{Ti}_{5 \mathrm{c}}$ interaction and protonation of surface $\mathrm{O}_{2 \mathrm{c}}$. This study contributes mechanistic insights into surface protonic conduction governed by adsorbed water layers on specific surfaces of polycrystalline oxides.

\section{Author contributions}

XK synthesized the samples, designed and performed the measurements, analyzed the data and wrote the original draft. TA and CN did the TEM measurements and in situ FTIR tests, respectively. XS contributed interpretation of data and revision of the manuscript. AC and TN conceived and supervised the study. All authors reviewed the manuscript.

\section{Conflicts of interest}

There are no conflicts to declare.

\section{Acknowledgements}

This work is enabled by a scholarship for XK by the China Scholarship Council (201806060141). Additional resources from collaboration within projects SUPROX (280868), and PH2ON (288320) financed by the Research Council of Norway are acknowledged.

\section{Notes and references}

1 R. Sato, S. Ohkuma, Y. Shibuta, F. Shimojo and S. Yamaguchi, J. Phys. Chem. C, 2015, 119, 28925-28933.

2 K. Xu, A. Chatzitakis and T. Norby, Photochem. Photobiol. Sci., 2017, 16, 10-16.

3 M. T. Colomer, Adv. Mater., 2006, 18, 371-374.

4 H. Ekström, B. Wickman, M. Gustavsson, P. Hanarp, L. Eurenius, E. Olsson and G. Lindbergh, Electrochim. Acta, 2007, 52, 4239-4245.

5 L. Liu, W. Chen and Y. Li, J. Membr. Sci., 2016, 504, 1-9.

6 D. Han and T. Uda, J. Mater. Chem. A, 2018, 6, 18571-18582.

7 M. I. Tejedor-Tejedor, F. M. Vichi and M. A. Anderson, J. Porous Mater., 2005, 12, 201-214.

8 E. L. Runnerstrom, G. K. Ong, G. Gregori, J. Maier and D. J. Milliron, J. Phys. Chem. C, 2018, 122, 13624-13635.

9 F. M. Vichi, M. I. Tejedor-Tejedor and M. A. Anderson, Chem. Mater., 2000, 12, 1762-1770.

10 F. Maglia, I. G. Tredici, G. Spinolo and U. AnselmiTamburini, J. Mater. Res., 2012, 27, 1975-1981.

11 S. Ø. Stub, K. Thorshaug, P. M. Rorvik, T. Norby and E. Vollestad, Phys. Chem. Chem. Phys., 2018, 20, 1565315660.

12 R. Mu, Z. J. Zhao, Z. Dohnalek and J. Gong, Chem. Soc. Rev., 2017, 46, 1785-1806.

13 S. Ø. Stub, E. Vøllestad and T. Norby, J. Phys. Chem. C, 2017, 121, 12817-12825.

14 O. Bikondoa, C. L. Pang, R. Ithnin, C. A. Muryn, H. Onishi and G. Thornton, Nat. Mater., 2006, 5, 189-192.
15 Y. He, A. Tilocca, O. Dulub, A. Selloni and U. Diebold, Nat. Mater., 2009, 8, 585-589.

16 L. Agosta, E. G. Brandt and A. P. Lyubartsev, J. Chem. Phys., 2017, 147, 024704.

17 A. Vittadini, A. Selloni, F. P. Rotzinger and M. Grätzel, Phys. Rev. Lett., 1998, 81, 2954-2957.

18 L. E. Walle, A. Borg, E. M. J. Johansson, S. Plogmaker, H. Rensmo, P. Uvdal and A. Sandell, J. Phys. Chem. C, 2011, 115, 9545-9550.

19 Q. Bai, M. Lavenas, L. Vauriot, Q. Le Trequesser, J. Hao, F. Weill, J. P. Delville and M. H. Delville, Inorg. Chem., 2019, 58, 2588-2598.

20 X. Kang, X.-Z. Song, S. Liu, M. Pei, W. Wen and Z. Tan, Nanoscale Adv., 2019, 1, 1372-1379.

21 N. Liu, Y. Chang, Y. Feng, Y. Cheng, X. Sun, H. Jian, Y. Feng, X. Li and H. Zhang, ACS Appl. Mater. Interfaces, 2017, 9, 5907-5915.

22 J. Wang, P. Zhang, X. Li, J. Zhu and H. Li, Appl. Catal., B, 2013, 134-135, 198-204.

23 F. Tian, Y. Zhang, J. Zhang and C. Pan, J. Phys. Chem. C, 2012, 116, 7515-7519.

24 Q. Shi, Y. Li, E. Zhan, N. Ta and W. Shen, CrystEngComm, 2015, 17, 3376-3382.

25 L. Jiang, L. Sun, D. Yang, J. Zhang, Y. J. Li, K. Zou and W. Q. Deng, ACS Appl. Mater. Interfaces, 2017, 9, 9576-9583.

26 F. Lin, Y. Chen, L. Zhang, D. Mei, L. Kovarik, B. Sudduth, H. Wang, F. Gao and Y. Wang, ACS Catal., 2020, 10, 42684279.

27 M. A. Henderson, Surf. Sci. Rep., 2002, 46, 1-308.

28 L. Mino, G. Spoto, S. Bordiga and A. Zecchina, J. Phys. Chem. C, 2012, 116, 17008-17018.

29 D. B. Asay and S. H. Kim, J. Phys. Chem. B, 2005, 109, 1676016763.

30 A. Tilocca and A. Selloni, Langmuir, 2004, 20, 8379-8384.

31 A. Tilocca and A. Selloni, J. Phys. Chem. B, 2004, 108, 47434751.

32 C. C. Pradzynski, R. M. Forck, T. Zeuch, P. Slavíček and U. Buck, Science, 2012, 337, 1529.

33 E. M. Kock, M. Kogler, B. Klotzer, M. F. Noisternig and S. Penner, ACS Appl. Mater. Interfaces, 2016, 8, 16428-16443.

34 C. Deiana, E. Fois, S. Coluccia and G. Martra, J. Phys. Chem. $C, 2010,114,21531-21538$.

35 G. Zhou and L. Huang, Mol. Simul., 2021, 47, 925-941.

36 R. Wang, M. L. Klein, V. Carnevale and E. Borguet, Wiley Interdiscip. Rev.: Comput. Mol. Sci., 2021, 11, e1537.

37 X. Lang, Y. Liang, J. Zhang, L. Li, L. Cao and H. Zhang, Phys. Chem. Chem. Phys., 2020, 22, 1371-1380.

38 P. Knauth and H. L. Tuller, J. Appl. Phys., 1999, 85, 897-902. 39 I. G. Tredici, F. Maglia, C. Ferrara, P. Mustarelli and U. Anselmi-Tamburini, Adv. Funct. Mater., 2014, 24, 51375146.

40 S. Erdal, C. Kongshaug, T. S. Bjørheim, N. Jalarvo, R. Haugsrud and T. Norby, J. Phys. Chem. C, 2010, 114, 9139-9145.

41 M.-H. Du, A. Kolchin and H.-P. Cheng, J. Chem. Phys., 2003, 119, 6418-6422. 
42 A. Schaefer, V. Lanzilotto, U. Cappel, P. Uvdal, A. Borg and A. Sandell, Surf. Sci., 2018, 674, 25-31.

43 S. Ø. Stub, E. Vøllestad and T. Norby, J. Mater. Chem. A, 2018, 6, 8265-8270.

44 A. S. Nowick and A. V. Vaysleyb, Solid State Ionics, 1997, 97, 17-26.
45 C. Sun, A. Selloni, A. Du and S. C. Smith, J. Phys. Chem. C, 2011, 115, 17092-17096.

46 T. R. Gordon, M. Cargnello, T. Paik, F. Mangolini, R. T. Weber, P. Fornasiero and C. B. Murray, J. Am. Chem. Soc., 2012, 134, 6751-6761.

47 Q. Huang, Y. Hu, G. He, C. Lin and C. Wei, Environ. Sci. Pollut. Res., 2018, 25, 35342-35351. 